\title{
Three-dimensional geometrical modelling of the femoral intramedullary cavity using ring cyclide model
}

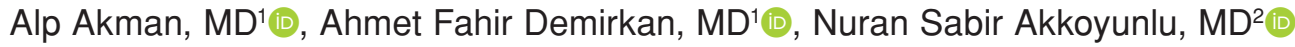 \\ 1'Department of Orthopedics and Traumatology, Faculty of Medicine Hospital, Pamukkale University, Denizli, Turkey \\ ${ }^{2}$ Department of Radiology, Faculty of Medicine Hospital, Pamukkale University, Denizli, Turkey
}

The femur has a natural bowing in the shaft region, which is unique in large bones. ${ }^{[1]}$ Any implant that will be put into femoral intramedullary cavity must be bowed. Any significant mismatch between the implant and femoral bowing may lead to complications..$^{[2-6]}$

The existing femoral shaft geometry studies are usually performed as a cross-sectional study ${ }^{[7]}$ or as a bowing measurement study. ${ }^{[8]}$ During the measurement of femoral bowing from the plain radiographs, the maximal femoral bowing axis is not always perpendicular to imaging direction. ${ }^{[9]}$ Hence, the measurements will be different than the actual values. On the other hand, cross-sectional studies are mostly related with structural anatomy. Threedimensional (3D) modelling allows very accurate determination of the maximal bowing plane. The 3D design method is virtually equivalent to the direct measurement of the bisected dried femur in vitro. ${ }^{[10]}$ During pre-study evaluations, we noticed that intramedullary structure of femur shaft have a close resemblance to a geometrical model called ring cyclide (Figure 1)..$^{[1]}$ For this purpose, the femoral shaft intramedullary cavity was resembled into a chord of a ring cyclide (Figure 2), where the rotational radius was centered approximately at its smallest

Received: March 05, 2019

Accepted: September 26, 2019

Published online: March 02, 2020

Correspondence: Ahmet Fahir Demirkan, MD. Pamukkale Üniversitesi Tıp Fakültesi Ortopedi ve Travmatoloji Anabilim Dalı, 20160 Pamukkale, Denizli, Türkiye.

E-mail: fahirdemirkan@yahoo.com

Doi: $10.5606 /$ ehc. 2020.65258

Citation: Akman A, Demirkan AF, Sabir Akkoyunlu N. Threedimensional geometrical modelling of the femoral intramedullary cavity using ring cyclide model. Jt Dis Relat Surg 2020;31(1):14-19.

\section{ABSTRACT}

Objectives: This study aims to investigate if geometrical modelling in addition to three-dimensional (3D) modelling will standardize models and allow performing mathematical calculations easily for the compatibility of femoral implant curvature.

Patients and methods: The study included 50 subjects $(27$ males, 23 females; mean age 55 years; range, 21 to 84 years). The femoral shaft intramedullary cavity was resembled into a chord of the ring cyclide, where the rotational radius was centered at its smallest radius. A 3D evaluation of the left femoral computed tomography data of the subjects was used to investigate the population parameters. The fitting was defined as being between the anterior and posterior border radii in the sagittal plane.

Results: The best fitting radius of implants was in between 90 to $99 \mathrm{~cm}$ in $72 \%$ of subjects in our sample. These radii values were lower than the mean intramedullary and cortical centerline radii which had only $62 \%$ and $50 \%$ fittings, respectively, among our population sample. The bowing radii and the smallest intramedullary width increased with the femoral length. These values were not affected by gender.

Conclusion: This modelling may have value for understanding femoral shaft intramedullary cavity geometry and may be a good tool to assess implant fitting.

Keywords: Anthropometry, bowing of the femur, humans, ring cyclide.

radius (Figure 3). Therefore, in this study, we aimed to investigate if geometrical modelling in addition to $3 \mathrm{D}$ modelling will standardize models and allow performing mathematical calculations easily for the compatibility of femoral implant curvature. ${ }^{[12]}$

\section{PATIENTS AND METHODS}

This study was conducted at Faculty of Medicine Hospital, Pamukkale University between November 2015 and April 2016. The femoral computed tomography (CT) scans which were taken before in our institution for another reason between January 2011 
and December 2011 were retrospectively evaluated for suitability. Inclusion criteria were (i) use of a standard protocol while taking CT scans (12 kV, $195 \mathrm{mAs})$, (ii) the whole femur scan including adjacent joints being in a single field of vision, (iii) a scanning resolution of $512 \times 512$ pixels, (iv) the scanning being sequential and in a standard slice thickness of $5 \mathrm{~mm}$ thick and $5 \mathrm{~mm}$ apart, and $(v)$ being aged more than 18 years. Exclusion criteria were excessive osteoporosis as a result of aging or previously taken chemotherapy or radiotherapy etc., previous operations, a present

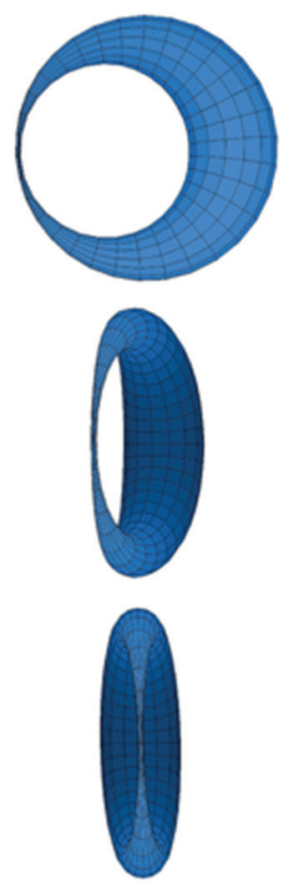

FIGURE 1. A ring cyclide's structure. (a)

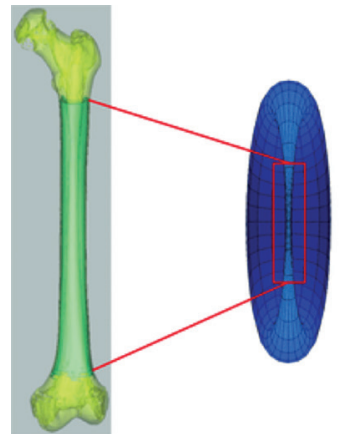

(b)

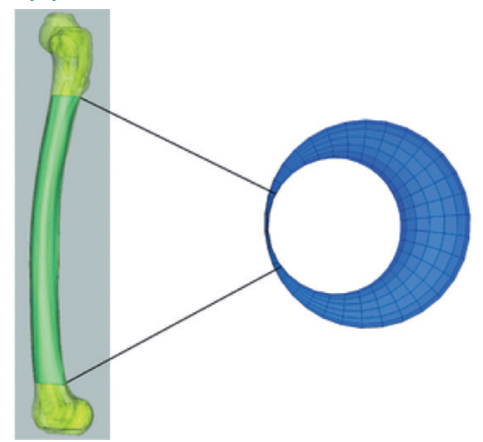

FIGURE 2. Structure of femoral shaft intramedullary cavity resembles a chord of ring cyclide. Three-dimensional whole bone model with superimposed shaft model and fitting ring cyclide in (a) coronal and (b) sagittal views. deformity, metallic objects/implants at the lower extremity and/or pelvis, any tumoral lesion and/or metastatic lesion in lower extremity and/or pelvis. After application of inclusion and exclusion criteria, 50 consecutive patients ( 27 males, 23 females; mean age 55 years; range, 21 to 84 years) were chosen for a 3D evaluation. The study protocol was approved by the Pamukkale University, Denizli, Turkey, Ethics Committee. A written informed consent was obtained from each subject. The study was conducted in accordance with the principles of the Declaration of Helsinki.

By using CT data, models were created with the Mimics version 10 (Materialise, Leuven, Belgium) 3D modelling program, as previously described. ${ }^{[2]}$ The minimum thresholds used were in accordance with findings of Kang et al. ${ }^{[13]}$ Two masks for each of the left femur were created to separate bones effectively. After that, a corresponding ring cyclide was virtually defined for each femur model (Figures 3, 4). The intramedullary and cortical centerline bowing radii were also calculated by using the sagittal centers of the radii calculation points, as previously described by Harma et al., ${ }^{[14]}$ however, the environment was 3D. The smallest coronal and sagittal intramedullary cavity widths were also found. Whole bone models were used to measure the axial femur length, which was the distance (a)

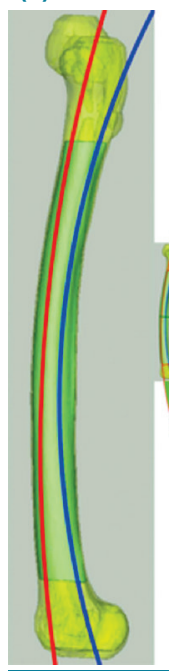

(b)

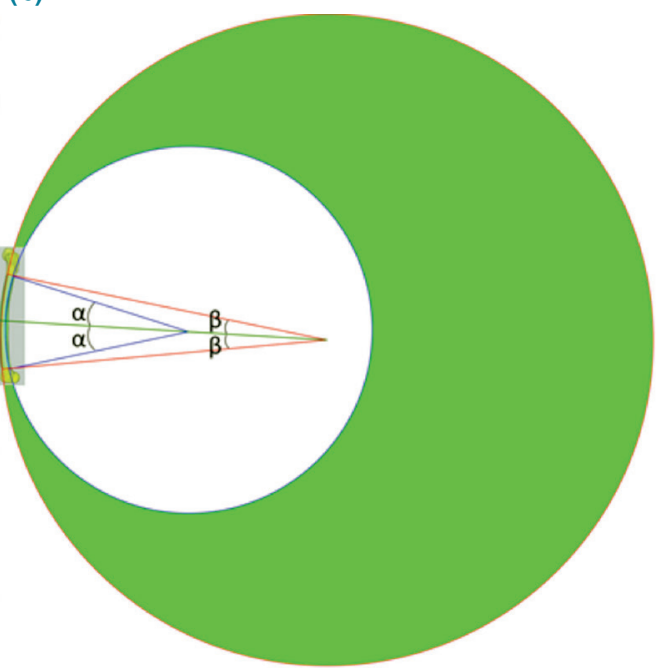

FIGURE 3. (a) Illustration of placement of anterior (blue circle) and posterior (red circle) border of fitting ring cyclide at its circular cross-section where rotational radius is centered -as opposing angles are same- approximately at its smallest. Green area illustrates fitting range in ring cyclide. (b) Closeup of fitting of borders. 

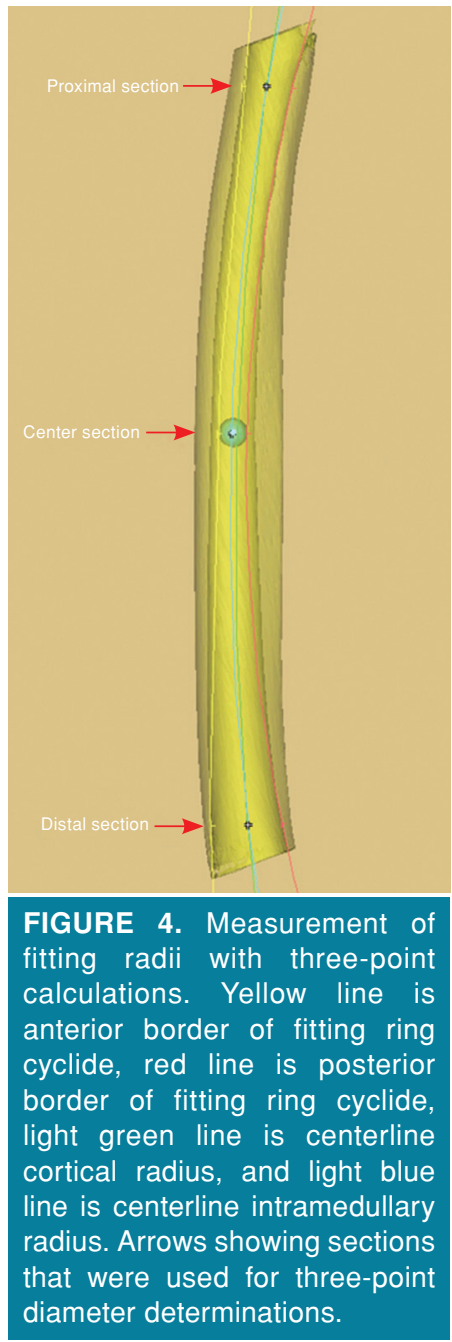

from the piriformis fossa to the intercondylar notch. The curved sagittal nail length and bowing angle were geometrically calculated as previously described by Harma et al. ${ }^{[14]}$ Each measurement was repeated six times per subject, and the highest and lowest readings were discarded. The average of the four measurements was used. The thickness measurements of this study resulted in an error range between $5.3 \%$ and $10.8 \%$, which is $3.2 \%$ and $7 \%$ for the best fitting radius. The groups were female and male femur models.

\section{Statistical analysis}

An independent samples t-test was used for the analysis of age- and gender-related data whereas a dependent samples paired t-test was used for the measurement of data by using Stata version 8 (Stata Corp., Texas, USA) software. The data were analyzed with a 95\% confidence interval and a $p<0.05$ was considered statistically significant. A priori power analysis was not performed; this was considered as a pilot study.

\section{RESULTS}

The bowing angles ranged between $12^{\circ}$ to $36^{\circ}$, with a mean of $22.2^{\circ}$. The mean values were not statistically significantly different between males and females $(\mathrm{p}=0.756)$.

The intramedullary centerline radius did not change with age, but the cortical centerline radius was decreased with age. Both radii did not differ by gender, but they were increased with an increase in the curved femoral length. The coronal and sagittal intramedullary cavity width were increased with an increase in the curved femoral length, but it did not alter with the changes to the intramedullary centerline radius ( $\mathrm{p}=0.868$ and $\mathrm{p}=0.283$, respectively). The sagittal width did not change with age, but the

\begin{tabular}{|c|c|c|}
\hline \multicolumn{3}{|l|}{$\begin{array}{c}\text { TABLE I } \\
\text { Summary of ages of subjects and } m\end{array}$} \\
\hline Parameter & Mean $\pm S D$ & Min-Max \\
\hline Age (year) & $55.2 \pm 14.6$ & $21-84$ \\
\hline Axial femoral length (cm) & $38.2 \pm 3.5$ & $26-45.6$ \\
\hline Bowing angle* (degrees) & $22.2 \pm 5$ & $12-36$ \\
\hline Curved femoral length* $(\mathrm{cm})$ & $38.6 \pm 3.6$ & $26.2-46$ \\
\hline The smallest intramedullary cavity width ${ }^{\star}(\mathrm{cm})$ & $13.7 \pm 2.6$ & $8-19.1$ \\
\hline The smallest coronal intramedullary cavity width $(\mathrm{cm})$ & $11.2 \pm 2.1$ & $6.7-15.2$ \\
\hline The anterior border radius ${ }^{\star}(\mathrm{cm})$ & $147.8 \pm 56$ & 73.3-288.6 \\
\hline The cortical centerline radius* $(\mathrm{cm})$ & $137.9 \pm 44.4$ & $68.8-262.5$ \\
\hline The intramedullary centerline radius* $(\mathrm{cm})$ & $107.4 \pm 28.9$ & 61.1-195 \\
\hline The posterior border radius* $(\mathrm{cm})$ & $78.7 \pm 19.4$ & $45.8-151.6$ \\
\hline
\end{tabular}




\begin{tabular}{|lccc|}
\multicolumn{4}{c}{ TABLE II } \\
Comparative $p$ values table for statistical analyses & \\
\hline Parameter & Age & Gender & Curved femoral length \\
\hline The smallest sagittal intramedullary cavity width & 0.089 & $<0.001^{*}$ & $<0.001^{*}$ \\
The smallest coronal intramedullary cavity width & $<0.001^{*}$ & $<0.001^{*}$ & $<0.001^{*}$ \\
The cortical centerline radius & $0.004^{*}$ & 0.336 & $0.027^{*}$ \\
The intramedullary centerline radius & 0.108 & 0.783 & $0.012^{*}$ \\
\hline${ }^{*}$ Accepted as statistically significant. & & & \\
\hline
\end{tabular}

coronal width was increased with age. Males had a larger cavity width in both measurements (Table I).

To suggest whether implant fitting at the mean intramedullary centerline radius was better or not, fitting of an implant with a radius of $107.4 \mathrm{~cm}$ was evaluated and the fitting was determined to be worse $(62 \%)(\mathrm{p}=0.024)$. The fitting at the mean cortical intramedullary centerline radius of $137.9 \mathrm{~cm}$ had much lower fitting $(50 \%)$, but was not different from the previous ( $\mathrm{p}=0.110)$ (Tables I and II). The best fitting implant radius was seemed to be around 90 to $99 \mathrm{~cm}$ in a fitting range analysis with $72 \%$ fitting. The fitting of the intramedullary centerline radius was worse than the best fitting range radii $(p=0.004$ for $90 \mathrm{~cm}$ and $\mathrm{p}=0.006$ for $99 \mathrm{~cm}$ ) (Figure 5 and Table III).

\section{DISCUSSION}

Our design showed that for defining a corresponding ring cyclide model of femoral intramedullary cavity, just three parameters are needed which are the anterior border radius, the posterior border radius and the smallest cavity width. This study also resulted in the establishment of two important parameters for

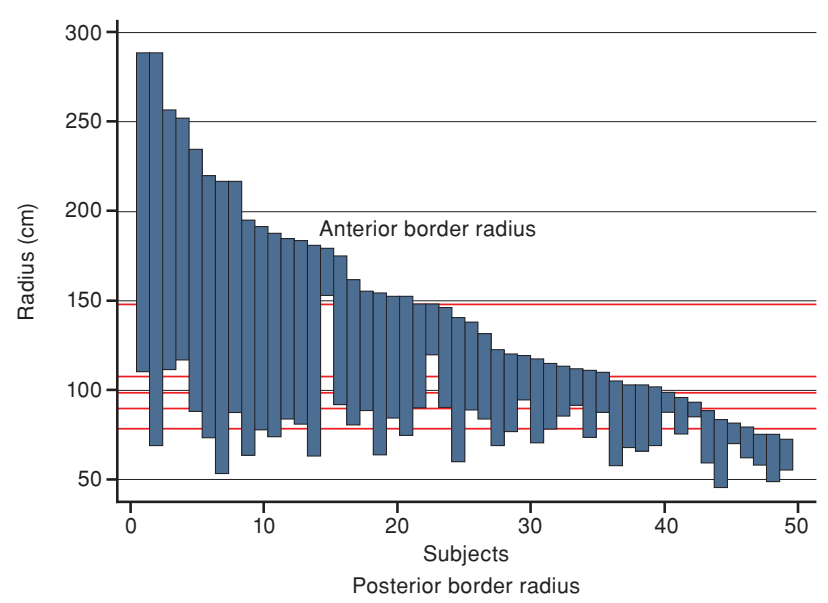

FIGURE 5. Comparative fittings between largest and smallest fitting radii of subjects: Fitting range analysis. (Subjects are in descending order according to anterior border radius.) curvature fitting analysis, which were the largest fitting radius (the anterior border radius) and the smallest fitting radius (the posterior border radius). The difference between them formed the fitting range for a person. As expected, the best fitting curvature was close to the mean of intramedullary bowing. However, the sample results in this study showed that the best fitting curvature was more bowed than that with a size of 90 to $99 \mathrm{~cm}$ but was limited to $72 \%$ of subjects in our sample. Femoral intramedullary cavity sizes have wide variation for optimal implant curvature fitting in the population and a single curvature size may not be sufficient (Figure 5).

The bowing radii (intramedullary or cortical) and the smallest intramedullary width (sagittal or coronal) were increased with the femoral length in this sample. These values were not affected by gender. The findings are comparable with those of Maratt et al. ${ }^{[8]}$ who postulate that femoral length is important for intramedullary bowing, while aging or gender have no effect. There is an increase in coronal intramedullary cavity width with aging; however, there is no statistically significantly different change in sagittal width. These findings are in concordance with those of Feik et al. ${ }^{[7]}$ who pointed out that aging causes a differential circumferential modeling and the effect of osteoporosis is mainly on the anterior cortex. The intramedullary bowing does not have a

\begin{tabular}{|ccc|}
\hline \multicolumn{3}{|c|}{ TABLE III } \\
Fitting ratios with different curvatures \\
\hline \multirow{2}{*}{ Radius $(\mathrm{cm})$} & \multicolumn{2}{c|}{ Fitting subject \& ratio } \\
\cline { 2 - 3 } & $\mathrm{n}$ & $\%$ \\
\hline 78.7 & 23 & 46 \\
90 & 36 & 72 \\
99 & 36 & 72 \\
107.4 & 32 & 62 \\
137.9 & 25 & 50 \\
147.8 & 21 & 42 \\
\hline
\end{tabular}


statistically significant correlation with aging, but the cortical bowing decreases similar to findings of Maratt et al. ${ }^{[8]}$ suggesting that there is no change of intramedullary bowing with aging.

The previously published results of femoral bowing range between 109 and $144 \mathrm{~cm} ;[3,4,6,15]$ however, there are differences in the measurement techniques. In a recent study performed by Maratt et ${ }^{2} .{ }^{[8]}$ the mean medullary radius of curvature of the femur was found as $110.4 \mathrm{~cm}$ in Caucasian Americans, which was 122.2 in African Americans and 101.1 in Asian Americans. The mean inner anterior cortex radius of curvature of the femur was found as $145.5 \mathrm{~cm}, 154.5 \mathrm{~cm}$ and $129.7 \mathrm{~cm}$, respectively. Our results were comparable with them, particularly with Caucasian Americans. In a study by Buford et al., ${ }^{[2]}$ in 19 cadaveric femur specimens (largely Caucasians) using a similar protocol to that used in this study, the anterior medullary canal radius of the curvature was shown to have a mean of $147.8 \mathrm{~cm}$ (calculated from given data) which is in accordance with this study. In a recent study with $3 \mathrm{D}$ design, Schmutz et al. ${ }^{[16]}$ found mean radius of curvature in the natural 3D antecurvature plane as $97.4 \mathrm{~cm}$ for Caucasians which was close to our findings $(107.4 \mathrm{~cm}$ in our study). However, a digital lateral radiogram work of Harma et al. ${ }^{[14]}$ have reported a value of $77.2 \mathrm{~cm}$ in Anatolian people which remains the lowest value reported before in the literature for Caucasians. ${ }^{[16]}$ In another study, Biçer et al. ${ }^{[17]}$ found a mean of 100 (one observer) and $120 \mathrm{~cm}$ (two observers) for anterior cortex curvature at 84 cadaveric femora of Anatolian people with no gender discrimination.

The established parameters for defining a corresponding ring cyclide model for a femoral intramedullary cavity can be calculated from the plain radiograms if magnification is standardized. However, the major problem will remain, which is the maximal bowing plane in that the circular crosssection of the ring cyclide lies. Its determination before taking radiograms will be very difficult particularly in subjects with coronal femoral bowing. However, 3D modelling allows accurate determination of the maximal bowing plane. The 3D modelling method is virtually equivalent to the direct measurement of the bisected dried femur in vitro. ${ }^{[14]}$

The limitations of this study are the subjective nature of reference point selection for the measurement of bowing radii and distances and its inherent error. ${ }^{[2]}$ Additionally, it is conceivable that reconstruction artefacts occur in the process of creating the 3D-CT models. ${ }^{[2]}$ The sample size of this study may be considered small for definitive conclusion. Furthermore, the sample was derived from $5 \mathrm{~mm}$-thick and $5 \mathrm{~mm}$-apart sequential CT data, which reduce the resolution.

In conclusion, the design of this study which is based on a ring cyclide model may have an important value for understanding femoral shaft intramedullary cavity geometry and may be a good tool to assess implant fitting for population studies. Furthermore, the method of modeling may lead to a new way of thinking in the interpretation of femoral geometry.

\section{Declaration of conflicting interests}

The authors declared no conflicts of interest with respect to the authorship and/or publication of this article.

\section{Funding}

The authors received no financial support for the research and/or authorship of this article.

\section{REFERENCES}

1. Walensky NA. A study of anterior femoral curvature in man. Anat Rec 1965;151:559-70.

2. Buford WL Jr, Turnbow BJ, Gugala Z, Lindsey RW. Threedimensional computed tomography-based modeling of sagittal cadaveric femoral bowing and implications for intramedullary nailing. J Orthop Trauma 2014;28:10-6.

3. Egol KA, Chang EY, Cvitkovic J, Kummer FJ, Koval KJ. Mismatch of current intramedullary nails with the anterior bow of the femur. J Orthop Trauma 2004;18:410-5.

4. Johnson KD, Tencer A. Mechanics of intramedullary nails for femoral fractures. Unfallchirurg 1990;93:506-11.

5. Roberts JW, Libet LA, Wolinsky PR. Who is in danger? Impingement and penetration of the anterior cortex of the distal femur during intramedullary nailing of proximal femur fractures: preoperatively measurable risk factors. J Trauma Acute Care Surg 2012;73:249-54.

6. Zuber K, Schneider E, Eulenberger J, Perren SM. Form and dimension of the bone marrow cavity of the human femur with reference to the fit of intramedullary implants. Unfallchirurg 1988;91:314-9.

7. Feik SA, Thomas CD, Bruns R, Clement JG. Regional variations in cortical modeling in the femoral midshaft: sex and age differences. Am J Phys Anthropol 2000;112:191-205.

8. Maratt J, Schilling PL, Holcombe S, Dougherty R, Murphy $\mathrm{R}$, Wang SC, et al. Variation in the femoral bow: a novel high-throughput analysis of 3922 femurs on cross-sectional imaging. J Orthop Trauma 2014;28:6-9.

9. Akman A, Demirkan F, Sabir N, Oto M, Yorukoglu C, Kiter E. Femoral bowing plane adaptation to femoral anteversion. Indian J Orthop 2017;51:49-54.

10. Kim JS, Park TS, Park SB, Kim JS, Kim IY, Kim SI. Measurement of femoral neck anteversion in 3D. Part 1: 3D imaging method. Med Biol Eng Comput 2000;38:603-9.

11. Weisstein EW. CRC Concise Encyclopedia of Mathematics. (2nd ed.). London: Taylor \& Francis/CRC, 2002, p.643. 
12. Atik OŞ. Every new technique either conservative or surgical is good? Eklem Hastalik Cerrahisi 2019;30:183-4.

13. Kang Y, Engelke K, Kalender WA. A new accurate and precise 3-D segmentation method for skeletal structures in volumetric CT data. IEEE Trans Med Imaging 2003;22:586-98.

14. Harma A, Germen B, Karakas HM, Elmali N, Inan M. The comparison of femoral curves and curves of contemporary intramedullary nails. Surg Radiol Anat 2005;27:502-6.

15. Harper MC, Carson WL. Curvature of the femur and the proximal entry point for an intramedullary rod. Clin Orthop Relat Res 1987;220:155-61.

16. Schmutz B, Kmiec S Jr, Wullschleger ME, Altmann M, Schuetz M. 3D Computer graphical anatomy study of the femur: a basis for a new nail design. Arch Orthop Trauma Surg 2017;137:321-31.

17. Biçer ÖS, Huri G, Tekin M, Mirioğlu A, Aydın A, Tan İ. Anatomic compatibility of femoral intramedullary implants: a cadaveric study. Acta Orthop Traumatol Turc 2016;50:222-6. 